\title{
Pulmonary Nocardiosis in a Kidney Transplant Recipient: A Case Report and Review of the Literature
}

Yaich S*, Charfeddine K, Zaghdane S, El Aoud N, Masmoudi M, Kharrat M and Hachicha J

Department of Nephrology, Hedi chaker Hospital Sfax, Tunisia

\begin{abstract}
Nocardiosis is a life threatening disease in solid organ transplant recipients. It is an uncommon but important infection for these patients. We report a case of 37- year-old kidney recipient who developed pulmonary nocardiosis that was successfully treated with intravenous imipenem and tetracyclines in conjunction with a reduction in immunosuppressive therapy. Four years later, graft function remains stable with complete regression of radiological abnormalities and absence of relapses. This case emphasizes the role of new potent immunosuppressants and diabetes in the occurrence of opportunistic infections. Nocardiosis should be suspected in the presence of pulmonary symptoms with unusual radiological presentation.
\end{abstract}

Keywords: Pulmonary nocardiosis; Renal transplantation; Immunosupression

\section{Introduction}

Nocardia is a ubiquitous aerobic actinomycete responsible for local or disseminated infection. The most frequent species associated with infections in humans are nocardia asteroids, nocardia brasiliensis, nocardia farscinica, and nocardia nova [1]. Nocardiosis is an opportunistic infection, occurring in upto $60 \%$ in deeply immunocompromised patients [2]. Clinical symptoms are non specific, depending on location [3]. Prognosis remains poor, especially in immunocompromised patients and disseminated forms with cerebronervous system involvement [4].

We report herein a case of pulmonary nocardiosis in a kidney transplant recipient that was successfully treated by imipenem and tetracycline.

\section{Case}

A 37- year-old man, with end stage renal disease secondary to an unknown nephropathy, received kidney transplantation on April 2005. The donor was his sister aged 34 years old. They had 3 HLA mismatch. The immunosupression consisted of thymoglobulin induction (5 days) followed by tacrolimus with through levels of $10-12 \mathrm{ng} / \mathrm{ml}$, with continuation of mycophenolate mofetil (MMF) and a steroid taper to $10 \mathrm{mg} / \mathrm{d}$. He was put on pneumocystis carinii prophylaxis of trimethoprin-sulfamethoxazole (TMP-SMX) for six months. The patient was discharged on the $15^{\text {th }}$ postoperative day with a good and stable renal function (serum creatinine of $80 \mu \mathrm{mol} / \mathrm{l}$ ). New onset diabetes occurred nine months later and was managed by oral anti diabetic drugs.

On March 2006, he was admitted to the internal medicine department for fever up to $40^{\circ} \mathrm{C}$, cough, anorexia and poor general health condition. Chest X-ray revealed a left hilar lesion consistent with infection in the context of the clinical history. He received a course of 10 - day of empiric therapy with cefotaxim $(2 \mathrm{~g} / \mathrm{d})$. However, his condition worsened and the pulmonary lesion appeared unresolved, so he was transferred to nephrology department.

Clinical examination revealed: normal blood pressure, dyspnoea of $32 \mathrm{cyc} / \mathrm{mn}$, oxygen saturation of $97 \%$ on room air and fever up to $39^{\circ}-40^{\circ} \mathrm{C}$

Lung examination revealed tachypnea and left basilar crackles. Cardiac, abdominal, skin and neurological examinations were with no remarks. Laboratory investigations revealed a serum creatinin of
$274 \mu \mathrm{mol} / \mathrm{l}$, white blood cell count $12600 / \mathrm{mm}^{3}$, elevated C -reactive -protein (194 mg/l), normal liver tests and metabolic acidosis $(\mathrm{pH}=$ 7.35 , serum bicarbonate level of $12 \mathrm{mmol} / \mathrm{l}$, oxygen saturation of $98 \%$ ). Urine and repeated blood culture were negative. Sputum culture was negative. Ultrasound echography was normal. Chest X-ray revealed a $5 \mathrm{~cm}$ para hilar nodular lesion with irregular contours (Figure 1). Thoracic CT scan revealed a large pseudo nodular lesion of the lower left field, extensive alveolar condensation with bronchogram in the left upper field surrounded by micro nodular lesions (Figure 2). An opportunistic infection and less probably a lung tumour were suspected. Bronchoalveolar lavage cultures revealed nocardia sensitive only for imipenem, amino glycosides and cyclines. An intravenous imipenem-based treatment was immediately started for six weeks, switched to oral doxycycline $200 \mathrm{mg}$ /day for six months more, with the complete resolution of the infection. Moreover, immunosuppressant drugs were temporarily reduced by decreasing the doses of tacrolimus with through levels of $7 \mathrm{ng} / \mathrm{ml}$ and switching mycophenolate mofetil to azathioprine. After six months of doxycycline oral treatment, the patient remains free of symptoms and with no evidence of relapse. The radiological examination shows complete resolution of the nodule (Figure 3). Five years later, there is no relapse and his transplant function is in a good state.

\section{Discussion}

Nocardiosis is a rare infection and difficult to diagnose. It is caused by a gram positive filamentous and strictly aerobic bacteria of the order Actinomycetales called "nocardia". Nocardia is frequently isolated from soils, dust, sand and stagnant water [5,6].

Infection occurs in severely immunocompromised patient with reduced-cellular mediated immunity such solid organ transplants, human-immunodeficiency virus infected patients, auto-immune diseases, neoplasia and chronic lung disease $[2,7]$. The most common

*Corresponding author: Soumaya Yaich, Route el Ain km 0.5, Department of Nephrology, Hedi Chaker Hospital Sfax, Tunisia, E-mail: soumayagfr@yahoo.fr

Received August 05, 2011; Accepted October 28, 2011; Published November 01, 2011

Citation: Yaich S, Charfeddine K, Zaghdane S, El Aoud N, Masmoudi M, et al (2011) Pulmonary Nocardiosis in a Kidney Transplant Recipient: A Case Report and Review of the Literature. J Transplant Technol Res 1:101. doi:10.4172/21610991.1000101

Copyright: () 2011 Yaich S, et al. This is an open-access article distributed under the terms of the Creative Commons Attribution License, which permits unrestricted use, distribution, and reproduction in any medium, provided the original author and source are credited. 
Citation: Yaich S, Charfeddine K, Zaghdane S, El Aoud N, Masmoudi M, et al. (2011) Pulmonary Nocardiosis in a Kidney Transplant Recipient: A Case Report and Review of the Literature. J Transplant Technol Res 1:101. doi:10.4172/2161-0991.1000101

Page 2 of 3

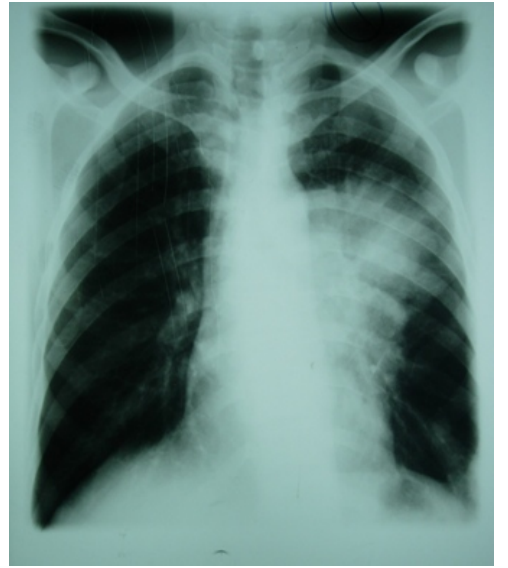

Figure 1: Chest X-ray revealed a $5 \mathrm{~cm}$ para hilar nodular lesion with irregular contours.
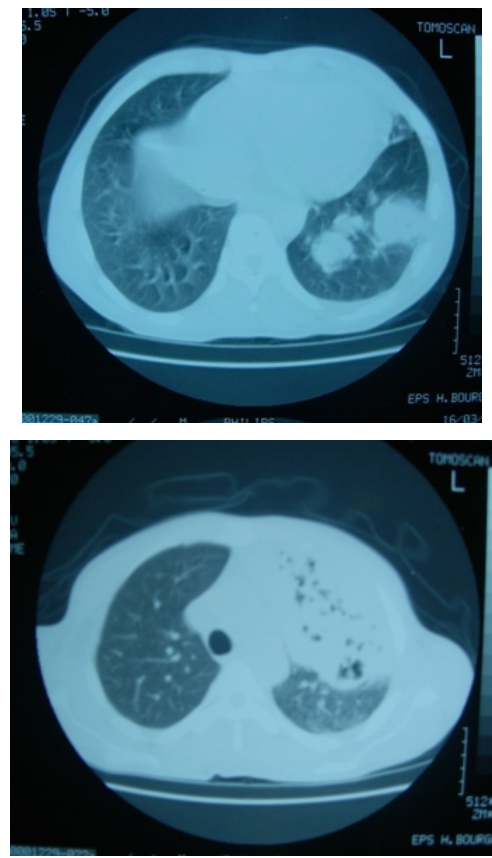

Figure 2: Thoracic CT scan revealed a large pseudo nodular lesion of the lower left field, extensive alveolar condensation with bronchogram in the left upper field surrounded by micro nodular lesions.

risk factors are corticosteroid therapy and immunosupression [7]. The overall incidence of nocardial infections in different immunosupressed patients ranges between $0.4 \%$ and $3.6 \%[2,8]$. In renal transplant recipients, this incidence ranges between 0.7 and $2.6 \%[4,2]$.

Newer potent immunosuppressive agents are main risk factors. So, long term corticoid treatment, MMF-tacrolimus association and use of depleting antibodies are risk factors for developing nocardiosis. In a recent matched case-control study of 35 solid organ transplant recipients with nocardiosis, risk factors that were identified are: receipt of high dose steroids, cytomegalovirus disease and a high median calcineurin inhibitor level in the preceding 30 days $(>15 \mu \mathrm{g} / \mathrm{ml}$ for tacrolimus and $>300 \mathrm{ng} / \mathrm{ml}$ for cyclosporine) [1].

Carnet et al observed only two cases of nocardiosis in 933 recipients transplanted between 1985 and 2002 and receiving cyclosporine, contrasting with four cases among 174 recipients transplanted between 1996 and 2002 and receiving Tacrolimus. Their data suggest that heavy immunosupression, and tacrolimus-based immunosupression are risk factors of nocardial infection [9].

Two other cases of pulmonary nocardiosis have been reported, respectively by Bilgarnia et al. and Flohr et al. [10,11]. The two recipients are sensitized with elevated panel reactive antibody, have retransplantation and underwent preconditioning using plasmapheresis and rituximab. The maintenance immunosuppressive regimen for the two patients was mycophenolate mofetil, tacrolimus, and steroids.

Our patient received induction treatment with thymoglobulin and was maintained on mycophenolate mofetil, steroids, and Tacrolimus. This association is deemed to be highly immunosuppressive. Moreover, diabetes mellitus is an additional risk factor favouring occurrence of opportunistic infection in our patient.

The infection is usually acquired through inhalation which results in a pneumonitis and eventually a dissemination of the infection, or through skin trauma [12]. There are three clinical forms: cutaneous, pulmonary and disseminated. The cutaneous form is related to the transcutaneous inoculation and is common in tropical and warm temperate areas. The most common mode of transmission remains inhalation, resulting in pulmonary localization.

The most frequent clinical presentation is a sub acute or chronic necrotizing pneumonia [12]. Pulmonary nocardiosis typically presents features made of dyspnoea, cough, chest pain, with no specific symptoms of fever, malaise and anorexia [13]. Radiological features are characterized by the presence of irregular nodular lesions, which may progress to cavitation.

They may also appear as diffuse pneumonic infiltrates or consolidate with pleural effusions [13]. We can find also alveolar syndromes, interstitial or reticulonodular infiltrates and even aspects of miliary and frequently excavated nodules or masses $[3,14]$. The main complication of this mode of transmission is haematogenous spread, resulting in a disseminated form of nocardiosis, defined by an involvement of two organs [14].

The organs most affected are the lungs, skin, subcutaneous tissue and cerebro nervous system. Other locations have been described: cardiac, ocular, osteoarticular [8].

Prophylaxis with cotrimoxazole is recommended in renal transplant

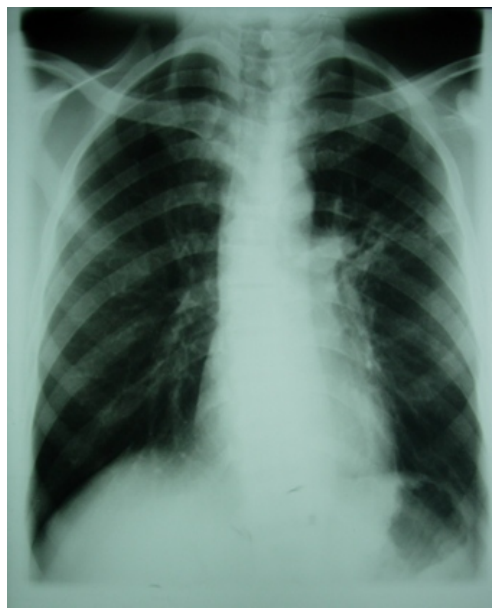

Figure 3: Resolution of the nodule on chest X-ray after six months therapy. 
patients for pneumocystis carini and urinary tract infection, and it is believed by some authors to protect from nocardia infection. However, there is increasing cases of breakthrough infections in patients taking TMP-SMX prophylaxis $[1,2,8]$. In fact, $14.2 \%$ of nocardial infections occur in patients while on cotrimoxazole prophylaxis [2].

Treatment relies on systemic antibiotic therapy. This latter should be adapted to the severity of the clinical picture, the different localization of the infection but also to the species.

Sulphonamides, mainly cotrimoxazole, are the treatment of choice $[3,14]$, although other molecules have proven their effectiveness, such as imipenem, amikacin, linelozid, cefotaxim, clarithromycin, ofloxacin and amoxicillin-clavulanic acid [15,16]. Linelozid, an oxazolidinone antibiotic which is gaining more attention, can be successfully used as an alternative to intravenous rout as well as primary therapy for nocardial infections at a dose of $600 \mathrm{mg}$ twice a day. However, this drug is not recommended for long-term therapy because of its serious side effects such as myelosupression, optic nerve damage and lactic acidosis $[8,17,18,19]$.

In the case of our patient, cefotaxim used as initial empiric therapy was ineffective. Thus, the sensitivity analysis of isolated germ to antibiotics confirms its resistance to this molecule and to TMP-SMX. Treatment with impenem relayed by tetracyclines was effective and well tolerated. Tetracyclines derivates are safe and effective. They may be considered as an alternative treatment for nocardia infections in transplanted patients. Leitersdorf et al treated ten transplanted patients (solid organ transplants and bone marrow transplants) with either doxycycline or minocycline. Nine out of the ten patients recover and one non compliant patient died of disseminated nocardiosis [20].

Duration of antibiotic therapy has not been well established and varies according to the authors, with a minimum of six months. Usually six to nine months in case of localized pulmonary are needed and nine to twelve months in case of cerebro nervous system involvement [9].

Surgery (drainage or removal) may be useful in cases of brain abscesses or tissue collections unresponsive to antibiotic therapy $[8,14]$. Reduction of immunosupression may be a helpful adjunctive therapy in severe forms of the disease but it is not a mandatory approach [8]. In our case, we choose to lighten the immunosuppressive therapy without stopping it, which was effective in controlling the infection.

Mortality remains high particularly in patients with disseminated nocardiosis. In pulmonary localization, mortality is about $40 \%$ and increases to $64 \%$ in disseminated nocardiosis and $100 \%$ in the presence of cerebro nervous system involvement [7].

\section{Conclusion}

Transplant physicians should be aware of this rare infection and consider nocardiosis in differential diagnosis of pneumonia especially with patients who have not responded to empiric treatment and when radiological features are atypical. Microbiological isolation is crucial for diagnosis, therefore bronchoalveolar lavage should be considered in patients with atypical pneumonia on radiological examination or with unusual clinical course under empirical treatment.

\section{References}

1. Peleg AY, Hussain S, Qureshi ZA, Silveira FP, Sarumi M, et al. (2007) Risk factors, clinical characteristics, and outcome of nocardia infection in organ transplant recipients: A matched case-control study. Clin Infect Dis 44: 13071314.

2. Minero MV, Marin M, Cercenado E, Rabadan PM, Bouza E, et al. (2009) Nocardiosis at the turn of the century. Medecine 88: 250-261.
3. Mootsikapun P, Intarapoka B, Liawnoraset W (2005) Nocardiosis in Srinagarin Hospital, Thailand: review of 70 cases from 1996-2001. Int J Infect Dis 9:154158

4. Queipo-Zaragoza JA, Broseta-Rico E, Alapont-Alacreu JM, Santos-Durantez M, Sanchez-Plumed J, et al. (2004) Nocardial infection in immunosuppressed kidney transplant recipients. Scand J Urol Nephrol 38:168-173.

5. Lerner PI (1996) Nocardiosis. Clin Infect Dis 22:891-903.

6. Laurent F, Mick V, Boiron P (1999) Clinique et biologie de la nocardiose. Annales de biologie clinique 57:545-554.

7. Martínez Tomás R, Menéndez Villanueva R, Reyes Calzada S, Santos Durantez M, Valles Tarazona JM, et al. (2007) Pulmonary nocardiosis: risk factors and outcomes. Respirology 12: 394-400.

8. Clark NM, AST Infectious Disesases Community of Practice (2009) Nocardia in solid organ Transplant Recipients. Am J Transplant 9: S70- S77.

9. Canet S, Garrigue V, Bismuth J, Chong G, Lesnik A, et al. (2004) Nocardiosisis it frequently observed after the introduction of new immunosuppressive agents in renal transplantation? Nephrologie 25: 43-48.

10. Biglarnia AR, Wadstrom J, Tufveson G, Eriksson BM (2008) Pulmonary nocardiosis with brain abscess in a sensitized kidney transplant recipient with a history of repeated graft loss and HLA- antibody depletion treatment- a case report. Ups J Med Sci 113: 111-116.

11. Flohr TR, Sifri CD, Brayman KL, Hagspiel KD, Sawyer RG, et al. (2009) Nocardiosis in a renal transplant recipient following rituximab preconditioning. Ups J Med Sci 114: 62-64.

12. Couraud S, Houot R, Coudurier M, Ravel AC, Coiffier B, et al. (2007) Infections pulmonaires à nocardia. Rev Mal Respir 24:353-357.

13. Fontana I, Gasloli G, Rossi AM, Bornacina C, Dodi F, et al. (2010) Nocardiosis in a kidney -Pancreas Transplant. J Transplant 2010: 1- 2.

14. Corti ME, Villafafie-Fioti MF (2003) Nocardiosis : a review. Int J Infect Dis 7 243-250.

15. Laurent F, Casoli E, Couble A, Boiron P (2001) Sensibilité aux antibiotiques des bactéries appartenant au genre Nocardia et traitement des nocardioses. Antibiotiques 3: 104-110.

16. Mooraki A, Jenabi A, Bastani B (2003) Resolution of pulmonary and cerebra nocardiosis in renal transplant patient despite continued immunosuppression: A case report. Transplant Proc 35: 2694-2695.

17. Lewis KE, Ebden P, Wooster SL, Rees J, Harrison GA (2003) Multi-system Infection with Nocardia farcinica-therapy with linezolid and minocycline. J Infect 46: 199-102

18. Brown-Elliott BA, Ward SC, Crist CJ, Mann LB, Wilson RW, et al. (2001) In vitro activities of linezolid against multiple nocardia species. Antimicrob Agents Chemother 45: 1295-1297.

19. Moylett EH, Pacheco SE, Brown-Eliott BA, Perry TR, Buescher ES, et al. (2003) Clinical experience with linezolid for the treatment of nocardia infection. Clin Infect Dis 36: 313-318.

20. Leitersdorf I, Silver J, Naparstek E, Raveh D (1997) Tetracycline derivates, alternative treatment for nocardiosis in transplanted patients. Clin Nephrol 48 48-51. 